\title{
A Vector Scale of Severity of Damages in Buildings
}

\author{
Félix Ruiz ${ }^{1}$, Antonio Aguado ${ }^{1}$ and Carles Serrat ${ }^{2}$ \\ ${ }^{1}$ Dept. of Civil and Environmental Engineering, Universitat Politècnica de Catalunya- \\ BarcelonaTECH, Jordi Girona, 1-3, 08034-Barcelona, Spain, felixruizgorrindo@gmail.com, \\ antonio.aguado@upc.edu \\ 2 IEMAE-EPSEB, Dept. of Mathematics, Universitat Politècnica de Catalunya-BarcelonaTECH, \\ Av. Dr. Marañón, 44-50, 08028-Barcelona, Spain, carles.serrat@upc.edu
}

\begin{abstract}
In the context of the existing buildings, along the recent years the concept of maintenance has changed from corrective maintenance to preventive maintenance, which is based in part on periodic inspections. There is ample evidence that preventive maintenance is more efficient than corrective maintenance, since severe deteriorations that may represent danger to people are avoided, and also money is saved. To make periodic inspections of the buildings is useful to quantify the extent to which deficiencies are severe or not, in order to facilitate decision making and prioritize therapeutic interventions. To this purpose many scales have been used and are used to assess the degree of gravity of the damages in constructive elements. But it is important to say that there is no common consensus and these scales are different between them according to the study to which they belong. Thus, the main goal of this article is to propose a methodology for calculating the degree of severity of damages in buildings, which is of widespread use. This calculation method, which is in distribution and in scalar, lets to calculate the severity index of systems and of the entire building, and it is easy to use and flexible.
\end{abstract}

Keywords: Scale, Severity Index, Damages, Direct Assignment Method, Quartiles.

\section{Introduction}

The rapid industrialization and population migration of the last 30 years, has led to a fast growing urbanization, doubling the building and partially the infrastructure stocks in very short periods (20-30 years) (Yang, 2006). In this context, the crucial indicator is the state of degradation of the different components of the stock (Kohler and Yang, 2007). Likewise, "What is not defined can not be measured. What is not measured can not be improved. What is not improved, it is always degraded". This phrase is from Sir William Thomson, Baron Kelvin of Largs. Although the phrase is from the nineteenth century, it is fully in force, and we are well aware of the importance of performing preventive maintenance in buildings in order to prevent their degradation and the appearance of severe deteriorations. In the framework of maintenance is true that to make periodic inspections of buildings, is useful to quantify the extent to which deteriorations are severe or not, in order to facilitate decision making and prioritize therapeutic interventions. In fact many scales have been used and are used to assess the degree of gravity of the constructive elements. But there is no common consensus and these scales are different from each other according to the study to which they belong (Ruiz, 2014).

All the referred shows the need to propose and validate a scale, in order to assess the severity index of constructive elements in buildings, which is of widespread use. Thus, the main goal of this article is to propose a calculation method, in distribution and in scalar, to calculate the degree of severity of systems and of the entire building, easy to use and flexible. 


\section{Methodology}

In most of the known scales, there is not any method to calculate the degree of severity of a system or of the entire building. In the few scales where there is a method, the output values are scalars, which do not show the variability of the distribution of severities. The proposed methodology in this paper aims to add the variability of the distribution of severities in the resulting vector scale. It is also remarkable to highlight that unlike what happens in other areas of science where there are scales widely used and commonly accepted, in the field of the buildings there is not a common scale for assessing the degree of severity of the constructive elements, and there are many different scales. There is no doubt that in building engineering would be very useful to have a common scale and a methodology widely used to assess the degree of severity of the buildings.

\subsection{Application Levels}

In this section the proposed methodology for calculating the degree of severity of damages in buildings as a whole (biggest proposed unit) or parts thereof, which will be called systems $(S)$ is introduced. It is considered appropriate to propose a reasonable division of building into systems, which is presented in Table 1. In order to provide flexibility to the methodology it allows that the total number of systems and the definition of them can be chosen by the technician that develops the study of the building, thus the proposed method is of general application. Therefore, the building as a whole is the sum of the systems that constitute it, as indicated in Equation (1)
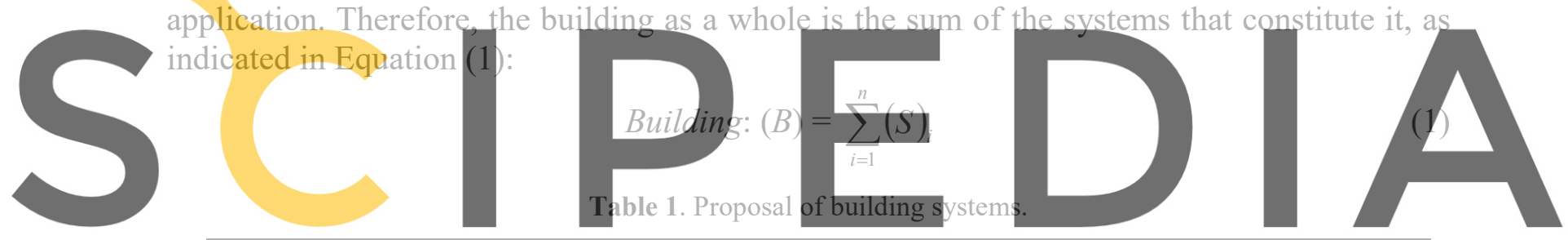

System Description Main constituent parts

Register for free at https//www.scipedia.com to download the version without the watermark

\begin{tabular}{|c|c|c|}
\hline 1 & Façades & $\begin{array}{l}\text { Claddings, base material, cantilevers, cornices, windows and other } \\
\text { practicable openings, railings, balustrades, ornamental elements, etc. }\end{array}$ \\
\hline 2 & Vertical structure & Pillars, load walls, foundation, etc. \\
\hline 3 & Horizontal structure & Beams, beam filling, vaults, arches, etc. \\
\hline 4 & $\begin{array}{l}\text { Roofs and inner } \\
\text { courtyards }\end{array}$ & $\begin{array}{l}\text { Roof tiles, pavements in flat roofs, waterproofing, thermal insulations, } \\
\text { skylights, walls and practicable openings for inner courtyards, etc. }\end{array}$ \\
\hline 5 & $\begin{array}{l}\text { Interior building } \\
\text { elements }\end{array}$ & $\begin{array}{c}\text { Partitions, interior walls, practicable openings, pavements, interior } \\
\text { claddings, etc. }\end{array}$ \\
\hline 6 & Staircases & Walls, stair structure, steps, railings, etc. \\
\hline 7 & Sewer facilities & Downpipes, drains, gutters, etc. \\
\hline 8 & Other facilities & Electricity, water, gas, elevators, etc. \\
\hline
\end{tabular}


Each system can be divided into zones which constitute the last and smallest proposed unit to value. A zone is defined as a specific part of a constructive element, as for example an area of a wood beams floor, an area of a façade, an area of a reinforced concrete beam floor, an area of a balcony, etc. Figure 1 shows some images that represent what is named zone.

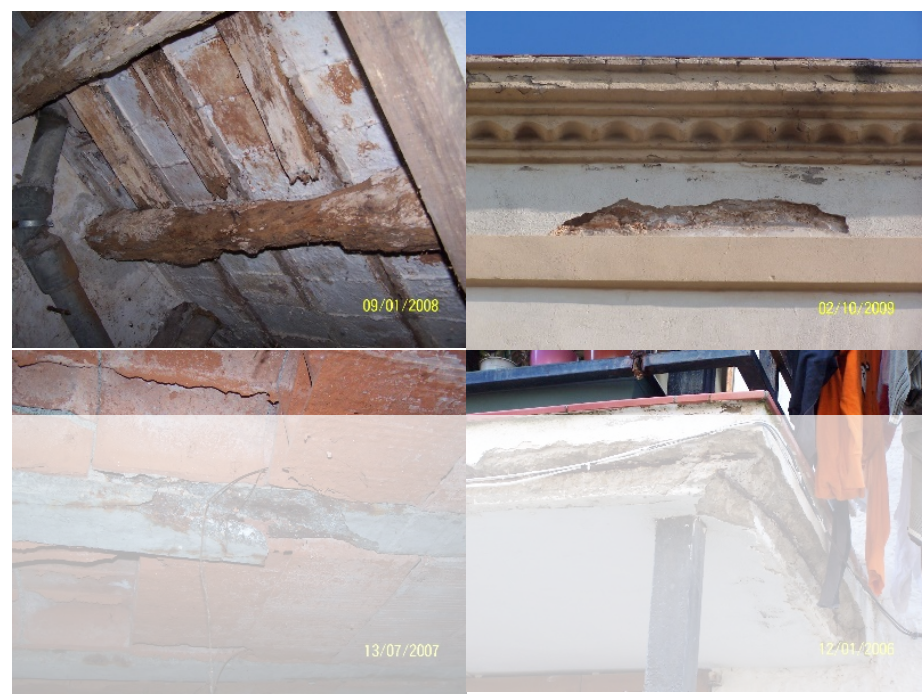

Figure 1. Images of four zones of construction elements.

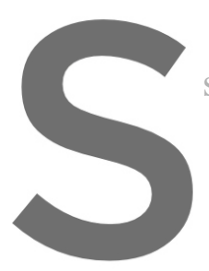

Thus, the set of zon

system, as shown in Equation (2):

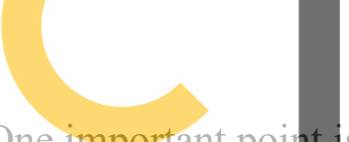

One important point is that this methodolog
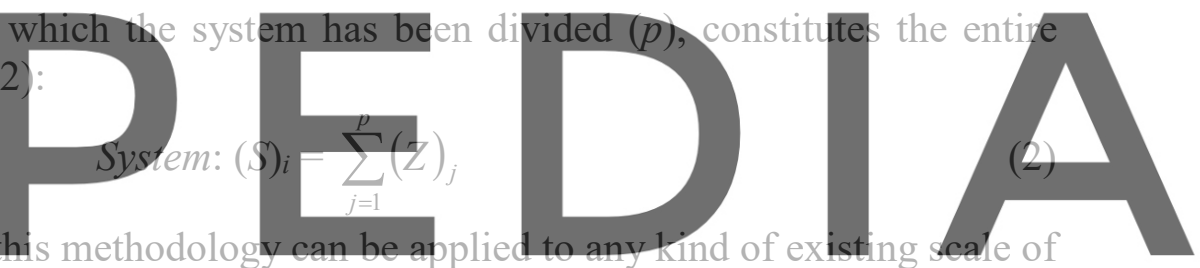

severity of damages in buildings, regardless they are based in direct assignment method (DA)

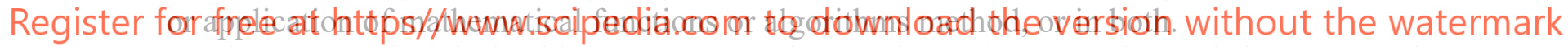

The proposed scale in this paper ranges from value 0 (zero gravity and the constructive element is in perfect condition) to value 10 (extreme gravity; it is not conceivable a greater gravity; pathology in terminal phase; collapse may occur at any time). Due to the scale is applicable to any type of constructive element (walls, beams, columns, bearing walls, façades, etc.), definitions are necessarily generic. These definitions can be seen in Ruiz et al. (2019).

\subsection{A Vector Scale of Severity of Damages in Buildings}

The proposed methodology, which is schematically presented in Figure 2, it is initiated by the direct assignment method (DA) based on the generic definitions of the reference scale, through which the grade or index of severity, $G$, is assigned to zones, $j$, of the building. In the variant called (a), the process ends at this level, which is in cases where the object of study is assessing the gravity of different zones of the building, but it is not necessary to evaluate the overall gravity of a system or of the whole building.

The next step of the methodology, when required, is to assess the severity of one or more systems, for which there are the variants called (b) and (c). In variant (b) DA method based on the generic definitions of the proposed scale is used, through which the degree of severity of 
the considered system is assigned. In variant (c) the calculation method that it is proposed in the next subsection is used, applying it to the $G_{j}$ values comprising the zones of the system, which allows to calculate the degree of severity of the considered system.

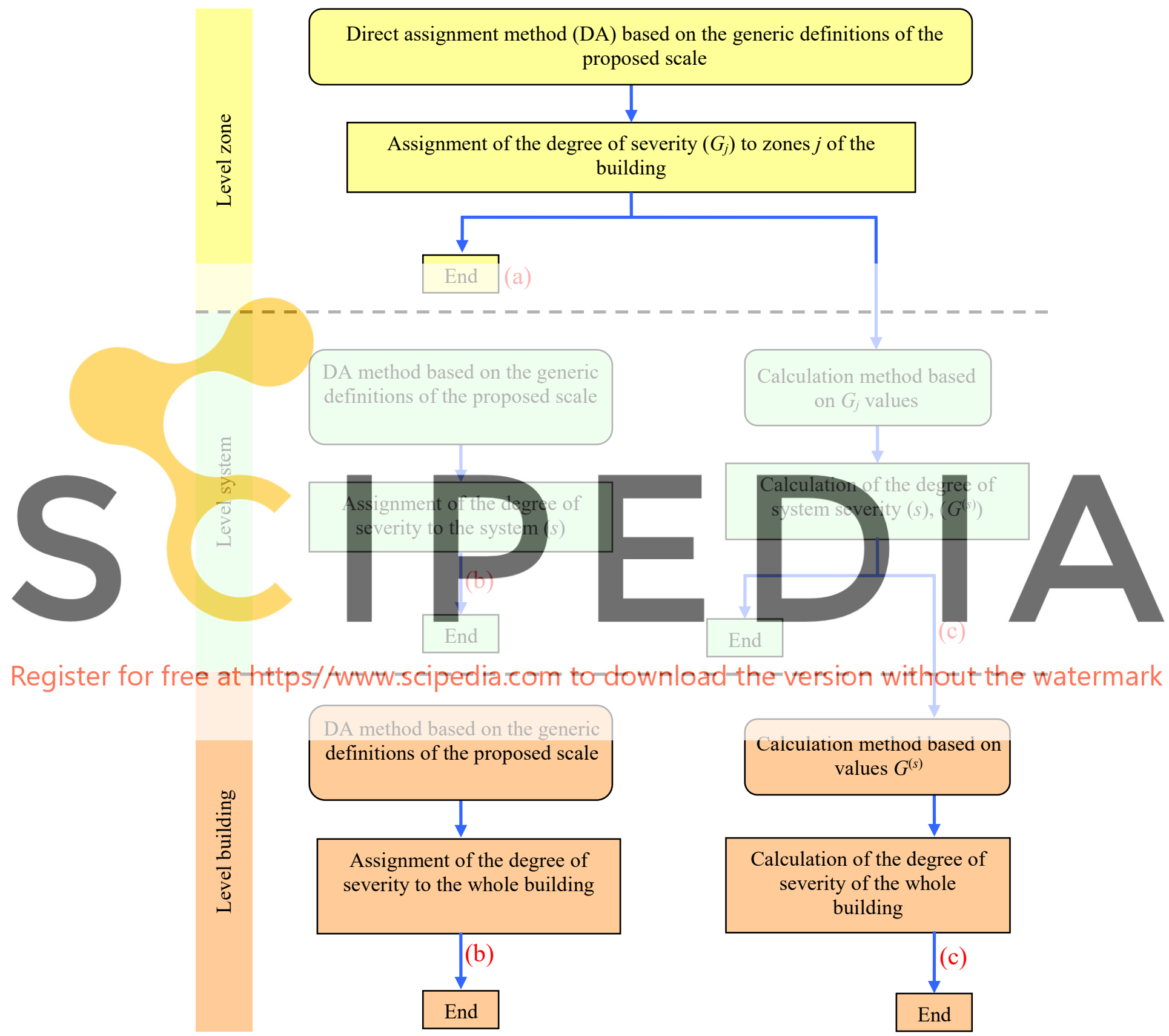

Figure 2. Methodology of application of the proposed scale

The variant (b) is of application in cases where the object of the study is such that it is necessary to spend a short time. Some of these cases may be the following: i) Global study of an urban area, in which it is necessary to assess the grade of gravity of hundreds or thousands of façades; ii) Emergency interventions, such as civil service technicians or firemen in cases of sever 
deteriorations with potential risk for people. The variant (c) will be of application in the remaining cases in which is required to determine the grade of gravity of a system or building.

\subsubsection{Definitions and computations at system level}

Within the framework of variant (c) in Figure 2, first it should be said that to obtain the resultant severity of a system based on the summation of the $G_{j}$ values of that system, applying a weight $w_{j}$ that is based on the area or proportion of the zone $j\left(A_{j}\right)$ regarding the area or whole unit $\left(A_{T}\right)$ of the considered system, gives adequate results only for cases that severity of the system is homogeneous, for example if the entire system is heavily degraded or everything is in good condition. However, it gives inadequate results in cases where there is a significant variability of severity in the system, especially in cases of extreme dispersion.

For this reason, after discarding the previous method of calculation for being inadequate, in order to calculate the resulting severity of a system $s$ it is proposed a method based on statistical quantiles. In this case quartiles are used as well as the minimum and maximum values of $G_{j}$ of the analyzed system, allowing to define the distribution of severity of system $s, G_{d}^{(s)}$, as follows in Equation (3):

$$
G_{d}^{(s)}=\left(q_{0}^{(s)}, q_{0.25}^{(s)}, q_{0.50}^{(s)}, q_{0.75}^{(s)}, q_{1.00}^{(s)}\right)
$$

where, $q_{0}^{(s)}$ and $q_{1.00}^{(s)}$ are the minimum and maximum value, respectively, of $G_{j}$ of the system $s$, and $q_{0.25}^{(s)}, q_{0.50}^{(s)}$ and $q_{0.75}^{(s)}$ are the maximum value of $G_{j}$ corresponding to the $25 \%, 50 \%$, $75 \%$ less degraded, respectively, of the system $s$.
With this methodology can be dirct)y observed the highest value of $G_{j}$ of each of the
evaluated systems of the building ( $q_{1}^{(s)}$ ). Similarly can be directly establish whether the
extension of the pathologies is generalized or ocalized in function of the $G_{j}$ values, which are
derived from analyzing the $25 \%, 50 \%$ or $75 \%$ of the system.

The resulting vector of a system $s$ allows to determine the priority of intervention of the

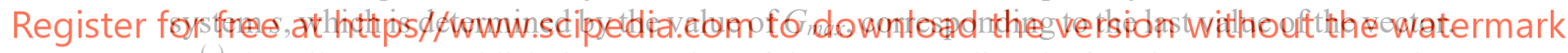
$G_{d}^{(s)}$ also allows to establish the extension of the corresponding dysfunction to $G_{\text {max }}$, extension that it is denoted by $e\left(., G_{\max }\right)$, that evaluates the position of the minimum quartile in which the $G_{\max }$ value appears. Formally, it is defined in Equation (4):

$$
e\left(G_{d}^{(s)}, G_{\max }\right)=q \text {, where } q \text { is the smallest } q_{k} \text { such that } q_{k}=G_{\max }
$$

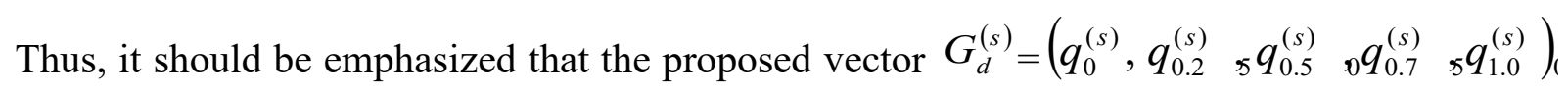
gives a lot of information about the severity of the system $s$, since besides it shows the minimum and maximum severity of the system, it shows the distribution of severities thereof. It must be said that while the vector $G_{d}^{(s)}$ describes numerically the grade of gravity of a system with sufficient accuracy, the distribution can be interesting to be summarized in a single value. To this end, we propose a method that transforms the vector $G_{d}^{(s)} \in\{0, \ldots 10\}^{5}$ into a scalar $G_{r}^{(s)} \in[0,10]$, which it is named resulting severity of the system, and it is denoted by $G_{r}^{(s)}$. 
To estimate the mean value of the distribution and using the relationship $E(X)=\int R(g) d g$ that calculates the expectancy of a positive random variable $X$ (in our case between 0 and 10) as the area under the complementary function of the distribution function $(R(g)=1-F(g))$, where $F$ denotes the distribution function, it is proposed that $G_{r}^{(s)}$ is obtained as the first order approximation of the area under the empirical distribution of $R$ from the distribution $G_{d}^{(s)}$. By construction it takes the values $R\left(q_{0}\right)=1-0=1 ; R\left(q_{0.25}\right)=1-0,25=0,75 ; R\left(q_{0.50}\right)=1-0,50$ $=0,50 ; R\left(q_{0.75}\right)=1-0,75=0,25$ y $R\left(q_{1.00}\right)=1-1=0$. Figure 3 illustrates this calculation for the numerical example $G_{d}^{(1)}=(0,2,5,5,8)$.
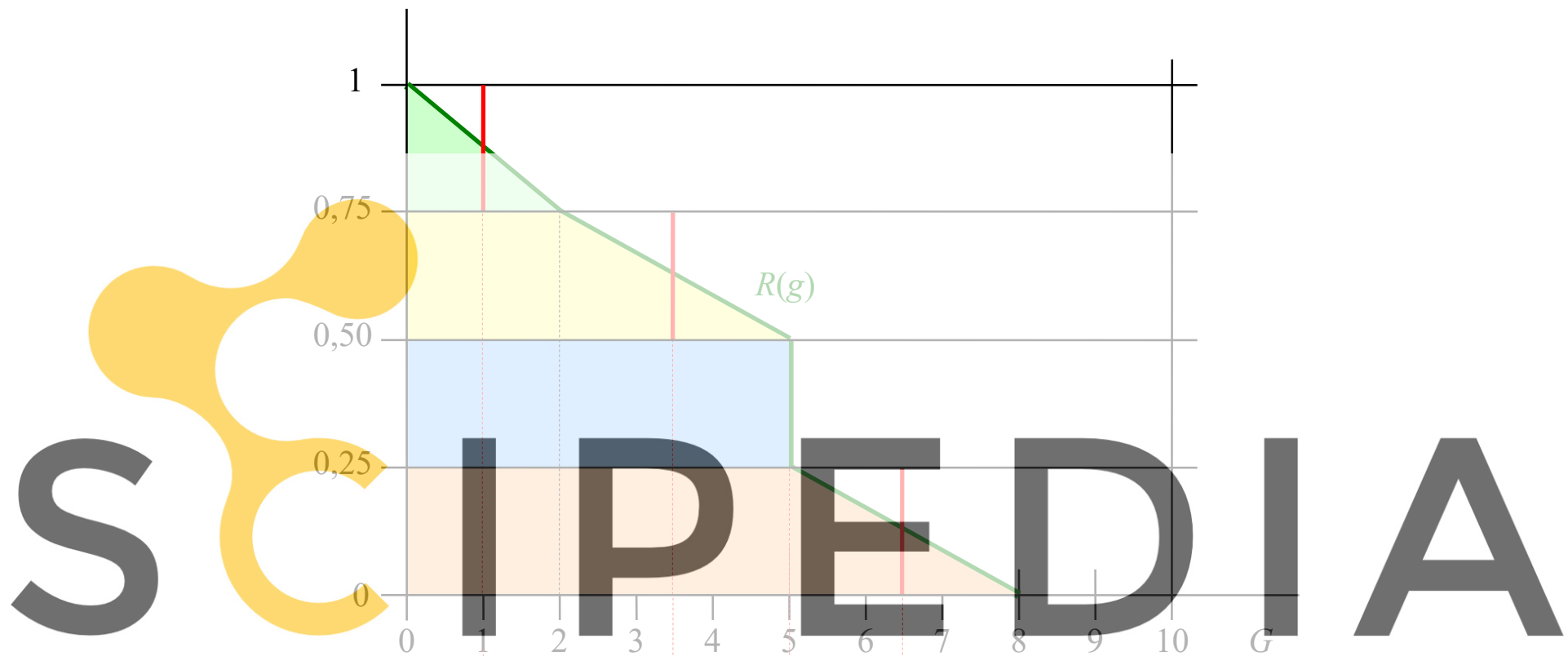

Register for free at https $\$$ / www.stipedia.com to todownload the version without the watermark

$$
m_{1}^{(s)} \quad m_{2}^{(s)} \quad m_{3}^{(s)} \quad m_{4}^{(s)}
$$

Figure 3. Function to determine the $G_{r}^{(s)}$ value.

So,

$$
\begin{aligned}
G_{r}^{(s)}=\left(\frac{q_{0}^{(s)}+q_{0.25}^{(s)}}{2}\right) \cdot 0,25 & +\left(\frac{q_{0.25}^{(s)}+q_{0.50}^{(s)}}{2}\right) \cdot 0,25+\left(\frac{q_{0.50}^{(s)}+q_{0.75}^{(s)}}{2}\right) \cdot 0,25+\left(\frac{q_{0.75}^{(s)}+q_{1.00}^{(s)}}{2}\right) \cdot 0,25= \\
& =\frac{q_{0}^{(s)}+2 \mathrm{q}_{0.25}^{(s)}+2 \mathrm{q}_{0.50}^{(s)}+2 \mathrm{q}_{0.75}^{(s)}+q_{1.00}^{(s)}}{8},
\end{aligned}
$$

or equivalently

$$
G_{r}^{(s)}=\sum_{i=1}^{4} \frac{m_{i}^{(s)}}{4},
$$

where $m_{i}^{(s)}$ are the midpoints between the components of $G_{d}^{(s)}$. 
In order to have a greater sensitivity over the parts of the building with greater severity, it is proposed to generalize the Equation (6), so it could be applied a set of coefficients $w_{i}^{(s)}$, which allows, among other possibilities, give more weight to the components on the right, which are those corresponding to the highest values $G_{j}$ of the system. In order to provide flexibility to the methodology, it is proposed that the technician can determine the relative weights $w_{i}^{(s)}$ to give to each coefficient, under the condition that $\sum_{i=1}^{4} w_{i}^{(s)}=1$. These coefficients act on the midpoints $m_{i}^{(s)}$ between components $q_{k}^{(s)}$, which allows to obtain $G_{r w}^{(s)}\left(G_{r}^{(s)}\right.$ weighted) defined as

$$
G_{r w}^{(s)}=\sum_{i=1}^{4} w_{i}^{(s)} \cdot m_{i}^{(s)}
$$

\subsubsection{Definitions and computations at building level}

\section{After obtaining vectors $G_{d}^{(s)}, s=1, \ldots, S$, representing the distribution of the gravity of each}

\section{system $s$, it is defined the distribution of severity of the building $\left(G_{d}^{*}\right)$, as follows:}

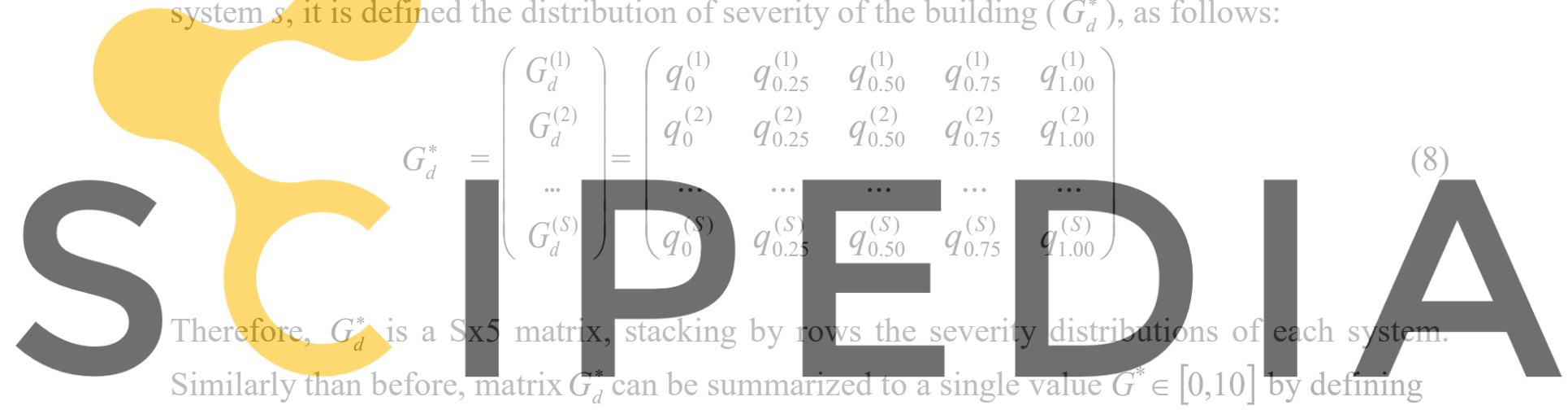

Register for free at https//www.scipedia.com to. download the version without the watermark

$$
G_{w}^{*}=\frac{\sum_{s=1}^{S} w^{(s)} \cdot w_{(\widetilde{s})}}{,}
$$

where

- $w^{(s)}$ : coefficient for each of the $S$ systems of the building, in order to give more weight to the most important systems.

- $\quad w_{(\widetilde{s})}$ : coefficient for each of the $S$ systems of the building, in order to give more weight to those systems that are in worse condition, which numerically means to give more weight to those highest values $G_{r w}^{(s)}$.

\section{Conclusion}

It should be noted that the proposed methodology can be easy applied by a technician. Just determining the $G_{j}$ severity (through DA method) and the $A_{j}$ surfaces of different areas $j$, it is possible to obtain automatically the resulting severities of each system $G_{r w}^{(s)}$ and the resulting severity of the building, by using a spreadsheet. Likewise, when applying the methodology to real cases, consistent results have 
been obtained, confirming the validation of the proposed methodology. Finally, it has been proven that this vector scale is an efficient tool as support to the technician in decision making.

\section{Acknowledgements}

This research has been partially supported by grants MTM2015-64465-C2-1-R (MINECO / FEDER) from the Ministerio de Economía y Competitividad (Spain) and 2017 SGR 622 from the Departament d'Economia i Coneixement de la Generalitat de Catalunya. Authors are grateful to members of the IEMAE, LABEDI and GRASS-GRBIO groups their valuable comments and suggestions in the development of the work.

\section{ORCID}

Felix Ruiz: http://orcid.org/0000-0002-7535-4490

Antonio Aguado: http://orcid.org/0000-0001-5542-6365

Carles Serrat: http://orcid.org/0000-0002-1504-5354

\section{References}

Kohler, N. and Yang, D. (2007). Long-term management of building stocks. Building Research and Information, 35(4), 351-362.

Ruiz, F. (2014). Escala de gravedad de daños en edificios: de la asignación directa a la constrastación estadística (in spanish). Doctoral Thesis. School of Civil Engineering of Barcelona. Polytechnical University of Catalonia.

Ruiz, F., Aguado, A., Serrat, C. and Casas J.R. (2019). Optimal metric for condition rating of existing buildings: is five the right number? Structure and Infrastructure Engineering, 15(6), 740-753, doi: https://doi.org/10.1080/15732479.2018.1557702.

Yang, D. (2006). International migration, remittances.

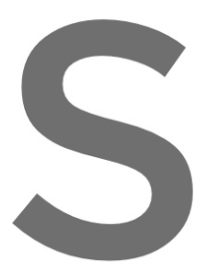
migrants exchange rate migrants exchange rate
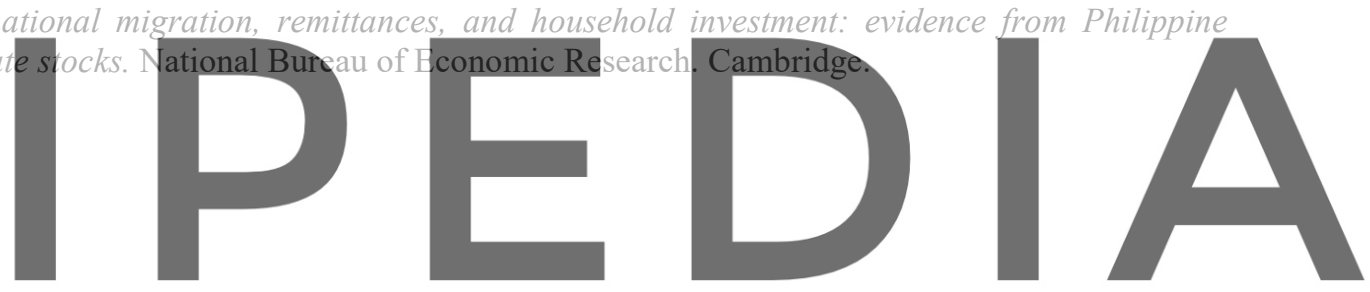

Register for free at https//www.scipedia.com to download the version without the watermark 